KUL-TF-07/13

arXiv:0708.2829 [hep-th]

\title{
Effective action for the field equations of charged black holes
}

\author{
Antoine Van Proeyen, Bert Vercnocke \\ Instituut voor Theoretische Fysica, Katholieke Universiteit Leuven, \\ Celestijnenlaan 200D B-3001 Leuven, Belgium.
}

\begin{abstract}
We consistently reduce the equations of motion for the bosonic $N=2$ supergravity action, using a multi-centered black hole ansatz for the metric. This reduction is done in a general, nonsupersymmetric setup, in which we extend concepts of BPS black hole technology. First we obtain a more general form of the black hole potential, as part of an effective action for both the scalars and the vectors in the supergravity theory. Furthermore, we show that there are extra constraints specifying the solution, which we calculate explicitly. In the literature, these constraints have already been studied in the one-center case. We also show that the effective action we obtain for non-static metrics, can be linked to the "entropy function" for the spherically symmetric case, as defined by Sen and Cardoso et al.
\end{abstract}

e-mails: antoine.vanproeyen@fys.kuleuven.be, bert.vercnocke@fys.kuleuven.be 


\section{Contents}

1 Introduction

2 Introducing our method: static metrics 4

2.1 Proposing an effective action . . . . . . . . . . . . . . . . 5

2.2 Reproducing the field equations from the effective action . . . . . . . . 7

2.3 The black hole potential . . . . . . . . . . . . . . . . . . . . . . . 9 9

3 Non-static metric $\quad \mathbf{1 0}$

3.1 Field equations . . . . . . . . . . . . . . . . . . . . . . . . . . . . . . . . . . . .

3.2 The effective action .......................... 11

4 Electric/magnetic duality $\quad 13$

4.1 Inequivalence of the 'original' and effective actions . . . . . . . . . . . . 14

4.2 Discussion and comparison to the literature . . . . . . . . . . . . . 15

5 Concluding remarks $\quad 16$ 


\section{Introduction}

It has been more than 10 years now since the attractor mechanism in supersymmetry was discovered, in the context of $N=2$ extremal black holes [1,2,3]. Since then, it has been realized that the attractor phenomenon is not restricted to the supersymmetry of the solution, but rather is a property related to the extremality of black holes. This was first proposed in [4], where also the concept of a black hole potential was introduced, and studied in detail later in [5,6,7]. In the latter, the non-extremal attractor equation was derived in great detail. All this work was done for static black holes.

In the context of string theory, the attractor phenomenon has also been well studied for BPS black hole systems [8]. In particular, it was shown [9, 10] that solutions exist with multiple centers. They can be described in the low energy limit as stable configurations of several coupled black holes. These no longer have to be described by a static metric.

We do not have the intention of giving a detailed description of the attractor phenomenon. We do, however, want to extend some of the ideas concerning the reduced action one generically writes down for such extremal black holes, which is the basis of a detailed study of the attractor equations. In the case of a static metric, a spherically symmetric metric ansatz is generically proposed - see for instance [4,7]. Using this ansatz, one eliminates the vectors through their equation of motion and integrates out angular dependence of the supergravity action. In this way one obtains a reduced action, governing the dynamics of the scalars, introducing a charge-dependent black hole potential along the way. For completeness, a (Hamiltonian) constraint has to be imposed on the system. Another method is based on integrating out angular dependence and a Legendre transform of the reduced Lagrangian is carried out. The stationary points of the entropy function thus obtained [11, 12] give the attractor equations. When one studies multicenter solutions, as is done in [9], one does not start from the standard form of the supergravity action exhibiting manifest four-dimensional covariance. Instead, one can derive the attractor behavior from a four-dimensional electric/magnetic duality invariant action. However, the author has not checked compatibility of this action with the full four-dimensional metric. When doing so, by comparing with the four-dimensional Einstein equations, constraints may have to be imposed on the system. In [9], this is avoided by immediately imposing BPS conditions on the reduced action. The BPS solutions are then supposed to satisfy these extra constraints.

We would like to obtain such an action in another way. We do this by studying the information contained in the Einstein equations. The Einstein equations, together with the equations of motion for the scalars and those of the vectors, govern the dynamics of the black hole solution. Knowing this, we will try to integrate (some of these) Einstein equations to give an effective action. The Einstein equations that cannot be derived from this effective action, will become constraints that have to be imposed on the solution. We will show that the effective action correctly describes the scalar and metric dynamics as well as those of

the vectors. In fact, the effective action we obtain in this way, is exactly the one that has already been studied [9], but now we also arrive at extra constraints that were not found then. We also show that the action we obtain is analogous to the concept of the entropy function of Sen [11] and Cardoso et al. [12], in the sense that our effective action is (minus) 
the entropy function for spherically symmetric metrics.

We apply our method to stationary spacetimes. Also, all the fields will be required to be time-independent. Both the static and non-static cases are discussed, in a nonsupersymmetric setup. This will allow us to propose an effective action, first for static but not necessarily spherically symmetric metrics. When we do look at spherical symmetry, we see that our effective action is the same as the reduced action in the literature. We also obtain the same constraint. However, the method we use is different from a dimensional reduction. There is a subtlety in the choice of independent variables. We will come back to this later. When we apply our machinery to non-static black hole systems, we arrive again at an effective action for the scalars, together with extra constraints. These constraints have not been found before. Also, we can straightforwardly generalize the concept of a black hole potential.

The outline of this paper is as follows. We begin by elaborating on results for a static black hole metric ansatz, in section 2, This will be more than just a review of what is known already for extremal black holes. We will bring our method into practice for a generic static metric ansatz, to establish the key features of our consistent reduction of the supergravity action by means of the field equations. This will lead us to proposing an effective action and a dynamical constraint restricting the solution. Where the original supergravity action had the scalars and the (four-dimensional) vectors as independent variables, this will no longer be the case here. The effective action reproduces the vector and scalar field equations, but only after a specific reparametrization of the vector contribution, in which we switch to new three-dimensional vector variables. Afterward, we make a specific choice of the metric and obtain indeed the same results as in the literature, including the notion of a black hole potential.

In section 3, we apply the same steps toward an effective action, this time for a general non-static metric ansatz admitting multicenter black holes. Again, the four-dimensional vectors will no longer be the independent variables in the effective action we obtain, but new three-dimensional ones will have to be considered. We will arrive at a generalization of the concept of a black hole potential. Furthermore, there will also be constraints generalizing those that we had for a static metric and we will present them in detail.

In section 4, we explain how the effective action we obtain is different from the supergravity action we started from. In fact, we start from a manifestly covariant action in four dimensions, which is not invariant under electric/magnetic duality rotations. The effective Lagrangian we obtain however, does transform as a scalar under electric/magnetic duality. But because we switched to three-dimensional gauge vectors as independent variables, it will no longer be covariant with respect to the full four-dimensional spacetime.

Finally, we conclude in section 5 .

\section{Introducing our method: static metrics}

Before applying our method to derive an effective action for multicenter black holes, we will look to static metrics. First, this will allow us to introduce the key ideas of our reduction of 
the equations of motion in a simpler setup. Secondly, we will be able to link the solutions we find to the results known in the literature. In particular, this will make it clear how we generalize the concept of a black hole potential, both in static configurations and later also in non-static ones.

A static spacetime metrid 1 has the general form:

$$
\begin{aligned}
& \mathrm{d} s^{2}=-\mathrm{e}^{2 U} \mathrm{~d} t^{2}+\mathrm{e}^{-2 U} \gamma_{m n} \mathrm{~d} x^{m} \mathrm{~d} x^{n}, \\
& \text { where: } \quad \partial_{t} U=\partial_{t} \gamma=0
\end{aligned}
$$

In the following, $\gamma_{m n}$ will be used to raise and lower indices and to define a covariant derivative $D_{m}$.

\subsection{Proposing an effective action}

We start from the bosonic part of the Einstein-Maxwell action coupled to Abelian vector fields:

$$
\begin{aligned}
S & =\int \mathrm{d}^{4} x \sqrt{g}\left(\frac{1}{2} R-g^{\mu \nu} G_{\alpha \bar{\beta}} \partial_{\mu} z^{\alpha} \partial_{\nu} \bar{z}^{\bar{\beta}}+\frac{1}{4}\left(\operatorname{Im} \mathcal{N}_{I J}\right) F_{\mu \nu}^{I} F^{\mu \nu J}-\frac{1}{8 \sqrt{g}}\left(\operatorname{Re} \mathcal{N}_{I J}\right) \varepsilon^{\mu \nu \rho \sigma} F_{\mu \nu}^{I} F_{\rho \sigma}^{J}\right) \\
& =S_{\text {Einstein }}+S^{(0)}+S^{(1)} .
\end{aligned}
$$

The independent variables in this action are the scalars $z^{\alpha}$, gauge vectors $A_{\mu}^{I}$, appearing through their fields strengths $F_{\mu \nu}^{I}=\partial_{\mu} A_{\nu}^{I}-\partial_{\nu} A_{\mu}^{I}$, and the metric $g_{\mu \nu}$. We have split the action in a gravity term $S_{\text {Einstein }}$, a scalar part $S^{(0)}$ and a vector part $S^{(1)}$. When seen in the context of a concrete $N=2$ supergravity theory, the scalar metric $G_{\alpha \bar{\beta}}$ will be hermitian and linked to the Kähler potential of the special geometry. The vector couplings are given by $\operatorname{Im} \mathcal{N}_{I J}$ and $\operatorname{Re} \mathcal{N}_{I J}$. The matrix $\mathcal{N}$ is determined by the special geometry and a choice of symplectic basis. The matrix $\operatorname{Im} \mathcal{N}$ will be negative-definite. For our purposes, however, the action may have both an arbitrary scalar metric $G_{\alpha \bar{\beta}}$ and arbitrary scalar-dependent vector couplings $\operatorname{Im} \mathcal{N}_{I J}$ and $\operatorname{Re} \mathcal{N}_{I J}$.

As explained in secetion 1, we would like to find an effective action starting from expressions for the Einstein equations. The Einstein tensor is defined as

$$
G_{\mu \nu}=2(\sqrt{g})^{-1} \frac{\delta S_{\text {Einstein }}}{\delta g^{\mu \nu}}=R_{\mu \nu}-\frac{1}{2} g_{\mu \nu} R .
$$

The Einstein equations are given by varying the action (2.2) with respect to the metric and yield:

$$
G_{\mu \nu}=T_{\mu \nu}
$$

\footnotetext{
1'Static' means that it admits a global, nowhere zero, timelike hypersurface orthogonal Killing vector field. A generalization are the 'stationary' spacetimes, which admit a global, nowhere zero timelike Killing vector field. In that case the components $g_{0 m}$ could be nonzero. We will turn first our attention to these static spacetimes.
} 
where $T^{\mu \nu}$ is the energy-momentum tensor. We have

$$
\begin{aligned}
& T_{\mu \nu}=T_{\mu \nu}^{(0)}+T_{\mu \nu}^{(1)} \\
& T_{\mu \nu}^{(0)}=-2(\sqrt{g})^{-1} \frac{\delta S^{(0)}}{\delta g^{\mu \nu}}, \quad T_{\mu \nu}^{(1)}=-2(\sqrt{g})^{-1} \frac{\delta S^{(1)}}{\delta g^{\mu \nu}} .
\end{aligned}
$$

Remember that our solution will be stationary and hence time derivatives vanish. Let us look first at the contribution from the scalar part of the action. We have

$$
T_{\mu \nu}^{(0)}=G_{\alpha \bar{\beta}}\left(-g_{\mu \nu} g^{\rho \sigma} \partial_{\rho} z^{\alpha} \partial_{\sigma} \bar{z}^{\bar{\beta}}+2 \partial_{\mu} z^{\alpha} \partial_{\nu} \bar{z}^{\bar{\beta}}\right) .
$$

For the vectors, we note that the metric only appears in the term with $\operatorname{Im} \mathcal{N}$ in the supergravity action (2.2). This leads to

$$
T_{\mu \nu}^{(1)}=\operatorname{Im} \mathcal{N}_{I J}\left(\frac{1}{4} g_{\mu \nu} F_{\rho \sigma}^{I} F^{J \rho \sigma}-F_{\mu \rho}^{I} F_{\nu \sigma}^{J} g^{\rho \sigma}\right) .
$$

When writing down the energy-momentum tensor, it proves useful to introduce the magnetic vectors

$$
F_{m}^{I}=\frac{1}{2} \gamma_{m n}(\sqrt{\gamma})^{-1} \varepsilon^{n p q} F_{p q}^{I}
$$

After some calculation, we see that the Einstein equations reduce to the following two equations:

$$
\begin{aligned}
& -\frac{1}{2} R_{m n}(\gamma)+\partial_{m} U \partial_{n} U+\partial_{(m} z^{\alpha} \partial_{n)} \bar{z}^{\bar{\beta}}-\mathrm{e}^{2 U} V_{m n}=0 \\
& D_{m} \partial^{m} U-\mathrm{e}^{2 U} \gamma^{m n} V_{m n}=0 .
\end{aligned}
$$

The tensor $V_{m n}$ is quadratic in the field strengths and given by the expression:

$$
V_{m n}=-\frac{1}{2} \operatorname{Im} \mathcal{N}_{I J}\left(\mathrm{e}^{-4 U} F_{0 m}^{I} F_{0 n}^{J}+F_{m}^{I} F_{n}^{J}\right)
$$

All this leads us to proposing the following effective action:

$$
S_{\text {eff }}=\int \mathrm{d} t \int \mathrm{d}^{3} x \sqrt{\gamma} \gamma^{m n}\left[-\partial_{m} U \partial_{n} U-G_{\alpha \bar{\beta}} \partial_{m} z^{\alpha} \partial_{n} \bar{z}^{\bar{\beta}}-\mathrm{e}^{2 U} V_{m n}\right] .
$$

The field equation of this action for $U$ is (2.10) if we keep $V_{m n}$ fixed during the variation. Of course, as $U$ does not appear in the scalar part of the action, the latter is not determined by this requirement. We will prove that the field equations for the scalars can also be derived from this effective action for a specific parametrization of $V_{m n}$. This will be clarified shortly. Only then will it be clear how to use this effective action. We want to emphasize that $\gamma^{m n}$ should not be seen as a dynamical variable in this action. Instead of its field equations as following from this effective action, we should take the Einstein equations from the original action (2.9) as constraints complementing the action (2.12). This will be explained below. 


\subsection{Reproducing the field equations from the effective action}

In the effective action, expression (2.11) is phrased in components of the field strengths $F_{\mu \nu}^{I}$. However, we can write it in terms of the symplectic vectors of field strengths and field equations. To do so, we introduce the following variables:

$$
\begin{aligned}
G_{I \mu \nu} & =\varepsilon_{\mu \nu \rho \sigma} \frac{\partial S}{\partial F_{\rho \sigma}^{I}} \\
& =\left(\operatorname{Re} \mathcal{N}_{I J}\right) F_{\mu \nu}^{J}+\frac{1}{2}\left(\operatorname{Im} \mathcal{N}_{I J}\right) g_{\mu \mu^{\prime}} g_{\nu \nu^{\prime}}(\sqrt{g})^{-1} \varepsilon^{\mu^{\prime} \nu^{\prime} \rho \sigma} F_{\rho \sigma}^{J} .
\end{aligned}
$$

Under electric/magnetic duality, $F^{I}$ and $G_{I}$ transform as a symplectic vector. If we denote the three-dimensional duals as a generalization of (2.8):

$$
F_{m}^{I}=\frac{1}{2} \gamma_{m n}(\sqrt{\gamma})^{-1} \varepsilon^{n p q} F_{p q}^{I}, \quad G_{I m}=\frac{1}{2} \gamma_{m n}(\sqrt{\gamma})^{-1} \varepsilon^{n p q} G_{I p q}
$$

we obtain from (2.13) and $\varepsilon^{0 n p q}=-\varepsilon^{n p q}$ (as we use $\varepsilon_{0123}=-\varepsilon^{0123}=1$ ):

$$
\left(\begin{array}{c}
F_{0 m}^{I} \\
G_{I 0 m}
\end{array}\right)=\mathrm{e}^{2 U} \Omega \mathcal{M}\left(\begin{array}{c}
F_{m}^{J} \\
G_{J m}
\end{array}\right)
$$

The matrices in the expression are the symplectic metric $\Omega$ and the matrix $\mathcal{M}$ defined by:

$$
\Omega=\left(\begin{array}{cc}
0 & \mathbb{1} \\
-\mathbb{1} & 0
\end{array}\right), \quad \mathcal{M}=\left(\begin{array}{cc}
-I-R I^{-1} R & R I^{-1} \\
I^{-1} R & -I^{-1}
\end{array}\right), \quad R=\operatorname{Re} \mathcal{N}, \quad I=\operatorname{Im} \mathcal{N} .
$$

These matrices contain indices $I$ and $J$ at appropriate positions automatically for (2.15) to make sense. This leads to

$$
\begin{aligned}
V_{m n} & =-\frac{1}{2} \mathrm{e}^{-2 U}\left(F_{m}^{I} G_{I 0 n}-F_{0 m}^{I} G_{I n}\right) \\
& =\frac{1}{2}\left(\begin{array}{ll}
F_{m}^{I} & G_{I m}
\end{array}\right) \mathcal{M}\left(\begin{array}{c}
F_{n}^{J} \\
G_{J n}
\end{array}\right) .
\end{aligned}
$$

$U$ does not appear in this expression for $V_{m n}$. This implies that if we consider $V_{m n}$ as a function of $F_{m}^{I}, G_{I m}$ and the scalars implicitly present in (2.16) and insert it as such in the effective action (2.12), then it is still valid that this action generates the right field equation for $U$. We now check that in this way it also generates the same scalar field equations as those obtained from $S^{(0)}$ and $S^{(1)}$ in the original action (2.2), where the vector fields $A_{\mu}^{I}$ were the other independent variables. Hence the field equations that should be reproduced are

$$
0=\partial_{\mu}\left(\sqrt{g} g^{\mu \nu} G_{\alpha \bar{\beta}} \partial_{\nu} \bar{z}^{\bar{\beta}}\right)+\frac{1}{4} \sqrt{g} \partial_{\alpha}\left(\operatorname{Im} \mathcal{N}_{I J}\right) F_{\mu \nu}^{I} F^{\mu \nu J}-\frac{1}{8} \partial_{\alpha}\left(\operatorname{Re} \mathcal{N}_{I J}\right) \varepsilon^{\mu \nu \rho \sigma} F_{\mu \nu}^{I} F_{\rho \sigma}^{J}
$$

where we denoted $\partial_{\alpha}=\frac{\partial}{\partial z^{\alpha}}$. Specifying metric (2.1) and the expressions for the field strengths in terms of $F_{m}^{I}$ and $G_{I m}$, this becomes

$$
0=\partial_{m}\left(\sqrt{\gamma} \gamma^{m n} G_{\alpha \bar{\beta}} \partial_{n} \bar{z}^{\bar{\beta}}\right)-\frac{1}{2} \mathrm{e}^{2 U} \sqrt{\gamma} \gamma^{m n}\left(\begin{array}{ll}
F_{m}^{I} & G_{m I}
\end{array}\right) \partial_{\alpha} \mathcal{M}\left(\begin{array}{c}
F_{n}^{J} \\
G_{n J}
\end{array}\right)
$$


where the indices $I$ and $J$ appear again in appropriate positions on the submatrices of $\partial_{\alpha} \mathcal{M}$. The latter are indeed the field equations for $z^{\alpha}$, obtained from the effective action

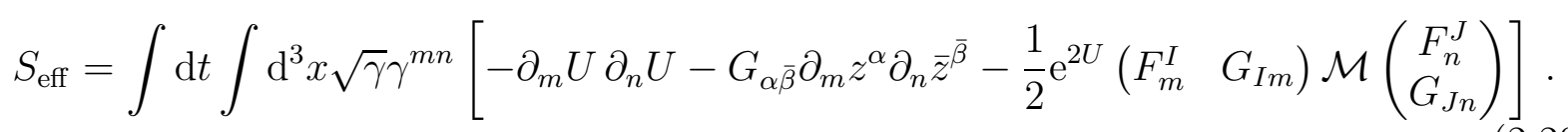

This action should be considered as an effective action for varying with respect to the variables $U, z^{\alpha}$ and $A_{m}^{I}, A_{I m}$, while $\gamma^{m n}$ should be considered as background. We saw already that the field equations of the original action for $\gamma^{m n}$ lead to constraint (2.9). We will now check what the field equations of the vector sector impose.

The Bianchi identities and the vector field equations from the four-dimensional action (2.2) with independent vectors $A_{\mu}^{I}$ are equivalent to

$$
\varepsilon^{\mu \nu \rho \sigma} \partial_{\nu}\left(\begin{array}{c}
F_{\rho \sigma}^{I} \\
G_{I \rho \sigma}
\end{array}\right)=0
$$

We can reproduce these equations from the effective action (2.20), if we now take threedimensional gauge vectors $A_{m}^{I}, A_{I m}$ as the fundamental vector variables. We consider then $F_{m n}^{I}$ and $G_{I m n}$ as the field strengths for these variables:

$$
F_{m n}^{I}=\partial_{m} A_{n}^{I}-\partial_{n} A_{m}^{I}, \quad G_{I m n}=\partial_{m} A_{I n}-\partial_{n} A_{I m}
$$

The Bianchi identities for the vectors $A_{m}^{I}, A_{I m}$ are given by

$$
\varepsilon^{m n p} \partial_{m}\left(\begin{array}{c}
F_{n p}^{I} \\
G_{\text {Inp }}
\end{array}\right)=0
$$

The components $F_{0 m}^{I}, G_{I 0 m}$ are no longer independent variables. Indeed, expression (2.15) should now be seen as their defining relation, showing how we can construct them from the three-dimensional vectors. Then the field equations for the vectors $A_{m}^{I}, A_{\text {Im }}$ obtained from varying the effective action (2.20) can be written as

$$
\varepsilon^{m n p} \partial_{n}\left(\begin{array}{c}
F_{0 p}^{I} \\
G_{I 0 p}
\end{array}\right)=0
$$

We see that, for stationary solutions, the vector field equations and Bianchi identities (2.21) are correctly reproduced by (2.23) and (2.24).

The effective action we propose (2.20) indeed reproduces all the field equations, with the following independent fields: scalars $U$ and $z^{\alpha}$, and three-dimensional vectors $A_{I m}, A_{m}^{I}$. To incorporate the full set of Einstein equations, constraint (2.9) still has to be considered. Note that we arrive at an action which is no longer covariant in four-dimensional spacetime, due to the vector term. We do however have an action that displays invariance under electric/magnetic duality. We come back to this in section 4 . 


\subsection{The black hole potential}

We now wish to establish the link with the black hole potential known in the literature. To do this, we try to find a solution to the equations (2.21). In terms of our preferred variables, this gives:

$$
\partial_{m} \sqrt{\gamma} \gamma^{m n}\left(\begin{array}{c}
F_{n}^{I} \\
G_{I n}
\end{array}\right)=0, \quad \partial_{[m} \mathrm{e}^{2 U} \mathcal{M} \Omega\left(\begin{array}{c}
F_{n]}^{I} \\
G_{I n]}
\end{array}\right)=0 .
$$

One way of solving these equations is to put

$$
\begin{aligned}
& F_{m}^{I}=\partial_{m} H^{I}, \quad G_{I m}=\partial_{m} H_{I}, \\
& \partial_{m} \sqrt{\gamma} \gamma^{m n} \partial_{n} H^{I}=\partial_{m} \sqrt{\gamma} \gamma^{m n} \partial_{n} H_{I}=0 .
\end{aligned}
$$

Remark that this brings us in the realm of supersymmetric solutions. Indeed, for instance in [9] it was shown that for BPS solutions the three-dimensional vectors are related to harmonic functions on $\mathbb{R}^{3}$ precisely in the manner (2.26).

We remain then with Bianchi identities of the form

$$
\left(\partial_{[m} \mathrm{e}^{2 U} \mathcal{M}\right) \Omega \partial_{n]}\left(\begin{array}{l}
H^{I} \\
H_{I}
\end{array}\right)=0
$$

which can be solved by assuming that all functions $\left(U\right.$, the scalars and the harmonic $H^{I}$ and $H_{I}$ ) depend only on one coordinate such that the $\partial_{m}$ and $\partial_{n}$ for $m \neq n$ in the above equation cannot both be non-vanishing. We denote this one coordinate as $\tau$. Thus, we have: $U(\tau), z(\tau), H^{I}(\tau)$ and $H_{I}(\tau)$.

A convenient metric is e.g. [13, 14]

$$
\gamma_{m n} \mathrm{~d} x^{m} \mathrm{~d} x^{n}=\frac{c^{4}}{\sinh ^{4} c \tau} \mathrm{d} \tau^{2}+\frac{c^{2}}{\sinh ^{2} c \tau}\left(\mathrm{d} \theta^{2}+\sin ^{2} \theta \mathrm{d} \varphi^{2}\right)
$$

Details on this parametrization are given in an appendix of [7]. This parametrization has the property $\sqrt{\gamma} \gamma^{\tau \tau}=\sin \theta$, which will be useful.

Harmonic means now just $\ddot{H}=0$, where a dot is a derivative w.r.t. $\tau$. So we can take

$$
H=\left(\begin{array}{l}
H^{I} \\
H_{I}
\end{array}\right)=\Gamma \tau+h, \quad h=\left(\begin{array}{l}
h^{I} \\
h_{I}
\end{array}\right), \quad \Gamma=\left(\begin{array}{c}
p^{I} \\
q_{I}
\end{array}\right) .
$$

We have here introduced the magnetic and electric charges in the symplectic vector $\Gamma$.

In this parametrization, the Lagrangian gets the form (up to a normalization):

$$
\mathcal{L}_{\text {eff }}=\dot{U}^{2}+G_{\alpha \bar{\beta}} \dot{z}^{\alpha} \dot{\bar{z}}^{\bar{\beta}}+\mathrm{e}^{2 U} V_{\mathrm{BH}}
$$

where the black hole potential is

$$
V_{\mathrm{BH}}=V_{\tau \tau}=\frac{1}{2} \Gamma^{T} \mathcal{M} \Gamma .
$$

The above is also the form of the reduced supergravity action, obtained by integrating out angular dependence, known in the literature (see e.g. [4,7]).

The one-dimensional effective Lagrangian (2.30) does not reproduce all of the field equations. Indeed, from the Einstein equations (2.9), we have to impose the following constraint:

$$
c^{2}-\dot{U}^{2}-G_{\alpha \bar{\beta}} \dot{z}^{\alpha} \dot{\bar{z}}^{\bar{\beta}}+\mathrm{e}^{2 U} V_{\mathrm{BH}}=0 .
$$




\section{$3 \quad$ Non-static metric}

Having established the key ideas in the last section, we would like to extend this now to the case where we have a more general, non-static ansatz for the metric. In [15,16] it was shown that time independent (BPS) configurations require a stationary metric that can be written in the form:

$$
\mathrm{d} s^{2}=-\mathrm{e}^{2 U}\left(\mathrm{~d} t+\omega_{m} \mathrm{~d} x^{m}\right)^{2}+\mathrm{e}^{-2 U} \gamma_{m n} \mathrm{~d} x^{m} \mathrm{~d} x^{n} .
$$

In fact, [15, 16] dictates $\gamma_{m n}=\delta_{m n}$. We will extend this slightly however, and allow $\gamma_{m n}$ to be an arbitrary metric on the three-dimensional space. We will proceed following the same steps as for a static metric. The main objective is to generalize the concept of the black hole potential, which was the tensor $V_{m n}$ (2.17) written down in a specific spherical coordinate system, and find the generalization of the extra constraint (2.9). We will keep using $\gamma_{m n}$ to raise an lower indices and to define a covariant derivative $D_{m}$. Again, we will make use of the dual field strengths (2.13). Remember, however, that this definition will lead to different expressions than in the static case, because of the explicit metric dependence in (2.13). Also, we define the three-dimensional duals as in (2.14).

In subsection 3.1 we give the field equations that have to be reproduced by the effective action we will construct. We calculate the Einstein equations and the field equations for the scalars and the vectors. We will later propose an effective action, in three-dimensional flat space. The function $U$ appearing in the metric will show up in this action as a scalar field; the functions $\omega_{m}$ behave as three-dimensional gauge vectors. Anticipating this, we will introduce the following field strength for $\omega_{m}$ :

$$
\Omega_{m n} \equiv \partial_{m} \omega_{n}-\partial_{n} \omega_{m}
$$

We will also find several constraints that have to be imposed on the fields in the effective action. Later, in subsection [3.2, we cast the effective action in a convenient form using variables $G_{I \mu \nu}$. We find in particular how the black hole potential can be generalized. The independent fields in the effective action will be the metric components $U$ and $\omega$, the scalars $z^{\alpha}$ and three-dimensional gauge vectors $A_{m}^{I}, A_{I m}$. It is then easy to show that the effective action correctly reproduces the field equations for the scalar fields and the vector field equations of the original supergravity action (2.2).

\subsection{Field equations}

We would like to construct an effective action, which correctly describes the dynamics of the $N=2$ supergravity theory, encoded in the action (2.2). Concretely, this means that we want to reproduce the scalar and vector field equations, and (part of) the Einstein equations. The metric ansatz we use now is the non-static one, given by (3.1). Again, we restrict ourselves to stationary solutions.

The scalar field equations for the supergravity action (2.2) are given by

$$
0=\partial_{\mu}\left(\sqrt{g} g^{\mu \nu} G_{\alpha \bar{\beta}} \partial_{\nu} \bar{z}^{\bar{\beta}}\right)+\frac{1}{4} \sqrt{g} \partial_{\alpha}\left(\operatorname{Im} \mathcal{N}_{I J}\right) F_{\mu \nu}^{I} F^{\mu \nu J}-\frac{1}{8} \partial_{\alpha}\left(\operatorname{Re} \mathcal{N}_{I J}\right) \varepsilon^{\mu \nu \rho \sigma} F_{\mu \nu}^{I} F_{\rho \sigma}^{J} .
$$


Expressing everything in terms of vectors $F_{m}^{I}$ and $G_{I m}$ using (2.14), this is equivalent to

$$
\begin{aligned}
0= & D^{m}\left(G_{\alpha \bar{\beta}} \partial_{m} \bar{z}^{\bar{\beta}}\right) \\
& -\frac{1}{2} \frac{\mathrm{e}^{2 U}}{\mathrm{e}^{4 U} \omega^{2}-1}\left[\left(\mathrm{e}^{4 U} \omega^{m} \omega^{n}-\gamma^{m n}\right)\left(\begin{array}{ll}
F_{m} & G_{m}
\end{array}\right) \partial_{\alpha} \mathcal{M}\left(\begin{array}{c}
F_{n} \\
G_{n}
\end{array}\right)\right] .
\end{aligned}
$$

The vector field equations and Bianchi identities on the other hand, can be written as

$$
\varepsilon^{\mu \nu \rho \sigma} \partial_{\nu}\left(\begin{array}{c}
F_{\rho \sigma}^{I} \\
G_{I \rho \sigma}
\end{array}\right)=0
$$

To find the Einstein equations, we start from the components of the Einstein tensor:

$$
\begin{aligned}
G_{00}= & \mathrm{e}^{4 U}\left(\frac{1}{2} R(\gamma)+2 D_{n} \partial^{n} U-\partial_{n} U \partial^{n} U+\frac{3}{8} e^{4 U} \Omega_{m n} \Omega^{m n}\right) \\
G_{m 0}= & \omega_{m} G_{00}-\frac{1}{2} D^{p}\left(\mathrm{e}^{4 U} \Omega_{m p}\right) \\
G_{m n}= & \omega_{m} \omega_{n} G_{00}-\omega_{(m} D^{p}\left(\mathrm{e}^{4 U} \Omega_{n) p)}\right) \\
& +\left(\gamma_{m n} \gamma^{p q}-2 \delta_{(m}^{p} \delta_{n)}^{q}\right)\left(-\frac{1}{2} R_{p q}(\gamma)+\partial_{p} U \partial_{q} U\right)-\frac{1}{8}\left(\gamma_{m n} \gamma^{p q}-4 \delta_{(m}^{p} \delta_{n)}^{q}\right) \mathrm{e}^{4 U} \Omega_{p r} \Omega_{q}{ }^{r} .
\end{aligned}
$$

To calculate the components of the energy-momentum tensor, we again make use of expressions (2.6) and (2.7), but now for the non-static metric ansatz (3.1). Again, we make use of magnetic vectors $F_{m}^{I}$ as defined in (2.14). We have a similar definition for $V_{m n}$ as in (2.11) and in addition, we define another symmetric tensor $A_{m n}$, quadratic in the field strengths:

$$
\begin{aligned}
V_{m n}= & -\frac{1}{2}\left(\operatorname{Im} \mathcal{N}_{I J}\right)\left(\mathrm{e}^{-4 U} F_{0 m}^{I} F_{0 n}^{J}+F_{m}^{I} F_{n}^{J}\right) \\
A_{m n}= & -\frac{1}{4}\left(\operatorname{Im} \mathcal{N}_{I J}\right)\left(\omega_{r} \omega^{r} F_{0 m}^{I} F_{0 n}^{I}+\omega_{m} \omega_{n} F_{0 r}^{I} F_{0}^{J r}-2 \omega_{p} \omega_{(m} F_{0}^{I p} F_{0 n)}^{I}\right) \\
& \left.-2 \varepsilon_{(m|p q|} \omega_{n)} F_{0}^{I p} F^{J q}-2 \varepsilon_{p(m \mid q} \omega^{p} F_{0 \mid n)}^{I} F^{J q}\right) .
\end{aligned}
$$

Imposing that each of the Einstein equations should vanish, we can in fact show that they are equivalent to the following set of independent equations:

$$
\begin{aligned}
0= & 2 D_{n} \partial^{n} U-2 \mathrm{e}^{2 U} \gamma^{m n}\left(V_{m n}+A_{m n}\right)+\frac{1}{2} \mathrm{e}^{4 U} \Omega_{m n} \Omega^{m n} \\
0= & \frac{1}{2} D^{r}\left(\mathrm{e}^{4 U} \Omega_{m r}\right)+\mathrm{e}^{2 U}\left(\operatorname{Im} \mathcal{N}_{I J}\right)\left(\omega_{m} F_{0 p}^{I} F_{0}^{J p}-\omega_{p} F_{0}^{I p} F_{0 m}^{J}-\varepsilon_{m p q} F_{0}^{I p} F^{J q}\right) \\
0= & \left(\gamma_{m n} \gamma^{p q}-2 \delta_{m}^{p} \delta_{n}^{q}\right)\left(-\frac{1}{2} R_{p q}(\gamma)+\partial_{p} U \partial_{q} U+\partial_{p} z^{\alpha} \partial_{q} \bar{z}^{\bar{\beta}}-\mathrm{e}^{2 U} V_{p q}\right) \\
& +\left(\gamma_{m n} \gamma^{p q}-4 \delta_{m}^{p} \delta_{n}^{q}\right)\left(\mathrm{e}^{2 U} A_{p q}-\frac{1}{8} \mathrm{e}^{4 U} \Omega_{p r} \Omega_{q}{ }^{r}\right) .
\end{aligned}
$$

Now we will propose an effective action that correctly describes the above dynamics.

\subsection{The effective action}

The effective action we propose will have the concise form

$$
S_{\text {eff }}=\int \mathrm{d} t \int \mathrm{d}^{3} x \sqrt{\gamma}\left[-\partial_{m} U \partial^{m} U-G_{\alpha \bar{\beta}} \partial_{m} z^{\alpha} \partial^{m} \bar{z}^{\bar{\beta}}+\frac{1}{8} \mathrm{e}^{4 U} \Omega_{m n} \Omega^{m n}\right]+\int \mathrm{d} t \int \mathrm{d}^{3} x \mathcal{L}_{\text {eff }}^{(1)} .
$$


Now we would like to cast the action (3.12) in a form similar to that we derived for the static solution. In particular, we want to extend the concept of a black hole potential we introduced there. To do this, we determine what the vector part of the effective action, $\mathcal{L}_{\text {eff }}^{(1)}$, should look like, to reproduce the scalar field equations (3.4) as well as the field equations and Bianchi identities for the vectors (3.5). First, we will choose as dynamical vector variables in the effective action (3.12) vectors $A_{m}^{I}, A_{I m}$, as was done in the static case, with

$$
F_{m n}^{I}=\partial_{m} A_{n}^{I}-\partial_{n} A_{m}^{I}, \quad G_{\text {Imn }}=\partial_{m} A_{\text {In }}-\partial_{n} A_{\text {Im }} .
$$

From (2.13), we obtain that the vectors $F_{0 m}^{I}$ are functions of the independent variables we have in the effective action (namely $U, \omega_{m}, z^{\alpha}$ and $A_{m}^{I}, A_{I m}$ ):

$$
\left(\begin{array}{c}
F_{0 m}^{I} \\
G_{I 0 m}
\end{array}\right)=\frac{\mathrm{e}^{2 U}}{\mathrm{e}^{4 U} \omega^{2}-1}\left[\left(\mathrm{e}^{4 U} \omega_{m} \omega_{n}-\gamma_{m n}\right) \Omega \mathcal{M}+(\sqrt{\gamma})^{-1} \gamma_{m q} \mathrm{e}^{2 U} \varepsilon^{n p q} \omega_{p} \mathbb{1}\right]\left(\begin{array}{c}
F_{n}^{J} \\
G_{J n}
\end{array}\right),
$$

where the indices $I$ and $J$ are suppressed on the matrices $(\Omega \mathcal{M})$ and $\mathbb{1}$. They appear in the different entries automatically in the appropriate positions.

The expression (2.17) for $V_{m n}$ in the static case, suggests we take the following expression as the vector-dependent part in the effective action (3.12):

$$
\begin{aligned}
(\sqrt{\gamma})^{-1} \mathcal{L}_{\text {eff }}^{(1)}= & \frac{1}{2}\left(F^{I m} G_{I 0 m}-G_{I}^{m} F_{0 m}^{I}\right) \\
= & -\frac{1}{2} \frac{\mathrm{e}^{2 U}}{\mathrm{e}^{4 U} \omega^{2}-1}\left[\left(\mathrm{e}^{4 U} \omega^{m} \omega^{n}-\gamma^{m n}\right)\left(F_{m}^{I} \quad G_{I m}\right) \mathcal{M}\left(\begin{array}{c}
F_{n}^{J} \\
G_{J n}
\end{array}\right)\right. \\
& \left.+2(\sqrt{\gamma})^{-1} \mathrm{e}^{2 U} \omega_{m} G_{I n} F_{p}^{I} \varepsilon^{m n p}\right] .
\end{aligned}
$$

Now we will calculate the field equations for the independent fields in the effective action (3.12), showing why the choice (3.15) is justified.

First, we can take a variation of the effective action w.r.t. the scalar field $U$. Using (3.14), we find

$$
\begin{aligned}
\frac{\delta \mathcal{L}_{\text {eff }}^{(1)}}{\delta U} & =\sqrt{\gamma} \mathrm{e}^{2 U} \operatorname{Im} \mathcal{N}_{I J}\left(F_{m}^{I} F^{J m}+\mathrm{e}^{-4 U} F_{0 m}^{I} F_{0}^{J m}+\Omega_{m n} F_{0}^{I m} F_{0}^{J n}-2(\sqrt{\gamma})^{-1} \varepsilon^{m n p} \omega_{m} F_{0 n}^{I} F_{p}^{J}\right) \\
& =-2 \sqrt{\gamma} \mathrm{e}^{2 U} \gamma^{m n}\left(V_{m n}+A_{m n}\right) .
\end{aligned}
$$

The functional derivative on the left-hand side has to be carried out when keeping the fields $\omega_{m}, z^{\alpha}$ and $A_{m}^{I}, A_{I m}$ fixed. When we now make a variation of the full effective action (3.12), we see that the field equation for $U$ is the same as the Einstein equation (3.9). Doing a likewise variation to find the field equations for $\omega_{m}$, keeping $F_{m}^{I}, G_{I m}$ fixed during variation, we reproduce expression (3.10). A variation w.r.t. $\gamma_{m n}$ gives inconsistency with the Einstein equations (3.9)-(3.11).

We now see that the effective action also generates the same scalar field equations as those obtained from the original action (2.2), where the vector fields $A_{\mu}^{I}$ were the other independent variables. Indeed, if we take the vectors $A_{m}^{I}, A_{I m}$ fixed during variation, the scalar field equations from the effective action (3.12) are exactly given by (3.4). 
Also the vector field equations and Bianchi identities (3.5) are reproduced. The Bianchi identities for gauge vectors $A_{m}^{I}, A_{I m}$ are given by

$$
0=\varepsilon^{m n p}\left(\begin{array}{c}
\partial_{m} F_{I n p} \\
\partial_{m} G_{I n p}
\end{array}\right) .
$$

Using (3.14) as a definition for $F_{0 m}^{I}$ and $G_{I 0 m}$, the field equations that follow from the effective action (3.12) are given by

$$
0=\varepsilon^{m p q} \partial_{p}\left(\begin{array}{c}
F_{0 q}^{I} \\
G_{I 0 q}
\end{array}\right) .
$$

We see that for stationary solution $\left(\partial_{0} F_{m n}=0\right)$, the field equations and Bianchi identities of the four-dimensional action (2.2) are correctly reproduced by (3.17) and (3.18).

Finally we note that the Einstein equations (3.11) cannot be reproduced from the effective action we propose. The conclusion is that they have to be supplied as additional constraints on the fields appearing in the effective action (3.12). They read

$$
\begin{aligned}
0= & \left(\gamma_{m n} \gamma^{p q}-2 \delta_{m}^{p} \delta_{n}^{q}\right)\left(-\frac{1}{2} R_{p q}(\gamma)+\partial_{p} U \partial_{q} U+\partial_{p} z^{\alpha} \partial_{q} \bar{z}^{\bar{\beta}}-\mathrm{e}^{2 U} V_{p q}\right) \\
& +\left(\gamma_{m n} \gamma^{r s}-4 \delta_{m}^{r} \delta_{n}^{s}\right)\left(\mathrm{e}^{2 U} A_{p q}-\frac{1}{8} \mathrm{e}^{4 U} \Omega_{p r} \Omega_{q}^{r}\right)
\end{aligned}
$$

where $V_{p q}$ and $A_{p q}$ where given in (3.7) and (3.8). The $F_{0 m}$ appearing in these terms has to be understood as a function of $F_{m}^{I}$ and $G_{I m}$ through relation (3.14).

We have seen that the effective action (3.12) correctly reproduces the field equations, for our non-static metric choice. It is important to note that the three-dimensional metric $\gamma_{m n}$ has to be considered as a background field in the effective action. Some of the Einstein equations cannot be reproduced - they have to be taken along as extra constraints. Again, the idea of four-dimensional gauge vectors has to be abandoned. The variables to be considered now are three-dimensional vectors $A_{m}^{I}, A_{I m}$. This implies that the effective action is no longer covariant. It does however remain invariant under electric/magnetic duality rotations. More on this in the following section. In the light of the discussion for non-static metrics, we also have a generalization of the concept of the black hole potential. It is given by the vector Lagrangian (3.15).

\section{$4 \quad$ Electric/magnetic duality}

We would like to show that the supergravity action (2.2) we started from is not equivalent to the effective action (3.12) we constructed. In fact, this can be seen from electric/magnetic duality. It is known that the Lagrangian (2.2) is not invariant under electric/magnetic duality. However, the effective action (3.12) is invariant.

In a first section, we show this in detail, while in a second subsection we establish a link with the work of Denef [9] and the entropy function as introduced in [11,12]. In particular, the effective action (2.30) we obtained for spherically symmetric metrics, gives exactly that entropy function. 


\subsection{Inequivalence of the 'original' and effective actions}

Non-static metric. On the one hand, we have the supergravity action (2.2):

$$
S=\int \mathrm{d}^{4} x \sqrt{g}\left(\frac{1}{2} R-g^{\mu \nu} G_{\alpha \bar{\beta}} \partial_{\mu} z^{\alpha} \partial_{\nu} \bar{z}^{\bar{\beta}}+\frac{1}{4}\left(\operatorname{Im} \mathcal{N}_{I J}\right) F_{\mu \nu}^{I} F^{\mu \nu J}-\frac{1}{8 \sqrt{g}}\left(\operatorname{Re} \mathcal{N}_{I J}\right) \varepsilon^{\mu \nu \rho \sigma} F_{\mu \nu}^{I} F_{\rho \sigma}^{J}\right)
$$

The Ricci scalar can be readily calculated from (3.6) and reads:

$$
\begin{aligned}
R & =-g^{\mu \nu} G_{\mu \nu} \\
& =\mathrm{e}^{2 U}\left(R(\gamma)-2 \partial_{m} U \partial^{m} U+\frac{1}{4} \mathrm{e}^{4 U} \Omega_{m n} \Omega^{m n}-2 \partial_{m} \partial^{m} U\right) .
\end{aligned}
$$

Again, we used the three-dimensional metric $\gamma_{m n}$ to raise and lower indices. We also use the expressions for $G_{I \mu \nu}(2.13)$ and for the vectors $F_{m}^{I}, G_{I m}$ (2.14) to rewrite the vector part of the Lagrangian:

$$
\begin{aligned}
\mathcal{L}^{(1)} & \equiv \frac{1}{4} \sqrt{g}\left(\operatorname{Im} \mathcal{N}_{I J}\right) F_{\mu \nu}^{I} F^{\mu \nu J}-\frac{1}{8}\left(\operatorname{Re} \mathcal{N}_{I J}\right) \varepsilon^{\mu \nu \rho \sigma} F_{\mu \nu}^{I} F_{\rho \sigma}^{J} \\
& =-\frac{1}{8} \varepsilon^{\mu \nu \rho \sigma} F_{\mu \nu} G_{\rho \sigma} \\
& =\frac{1}{2} \sqrt{\gamma}\left(F^{I m} G_{I 0 m}+G_{I}^{m} F_{0 m}^{I}\right)
\end{aligned}
$$

Specifying the metric and restricting ourselves again to stationary solutions, the supergravity action is, up to a total derivative, given by

$$
S=\int \mathrm{d}^{4} x \sqrt{\gamma}\left[\frac{1}{2} R(\gamma)-\partial_{m} U \partial^{m} U+\frac{1}{8} \mathrm{e}^{4 U} \Omega_{m n} \Omega^{m n}-G_{\alpha \bar{\beta}} \partial_{m} z^{\alpha} \partial^{m} \bar{z}^{\bar{\beta}}\right]+\int \mathrm{d}^{4} x \mathcal{L}^{(1)}
$$

This action is not the same as the effective action we obtained earlier. First, it has to be understood in a different way - the reduced action (4.4) has as independent variables $U, \omega$, the scalars $z^{\alpha}$ and four-dimensional gauge vectors $A_{\mu}^{I}$. In this way it reproduces the field equations of the scalars and the vectors, and the Einstein equations (3.9) and (3.10). Again, (3.11) has to be taken along as an extra constraint. But more importantly, it is clear that the vector Lagrangian is not invariant under electric/magnetic duality.

This is clearly different from the effective action (3.12) we obtained. There we had instead of $\mathcal{L}^{(1)}$ the following vector part in the Lagrangian:

$$
\mathcal{L}_{\text {eff }}^{(1)}=\frac{1}{2} \sqrt{\gamma}\left(F^{I m} G_{I 0 m}-G_{I}^{m} F_{0 m}^{I}\right)
$$

the independent vector variables being three-dimensional gauge vectors $A_{m}^{I}$ and $A_{I m}$. This expression clearly is invariant under electric/magnetic duality rotations.

Static metric. When we use the metric ansatz (2.1), we can carry through the same discussion, with $\omega_{m}=0$. 


\subsection{Discussion and comparison to the literature}

The expression (4.5) is truly invariant under electric/magnetic duality rotations. This shows that our method of constructing an effective action leads us from a form (4.3) which makes general covariance manifest, but lacking electric/magnetic invariance, to a form (4.5) which incorporates the latter but misses out on four-dimensional covariance. In fact, a manifestly covariant four-dimensional action invariant under electric/magnetic duality does not exist. In 9], Denef comments on this. Because he works in the framework of type IIB compactification, the problem is equivalent to the non-existence of a straightforward generally covariant action for the self-dual four-form potential. Based on the work of [17, 18], he constructs a fourdimensional action which exhibits invariance under electric/magnetic duality rotations. Our effective action is in fact the same as his, up to a normalization of the vectors. However, in his work constraints (3.19) were not taken into account.

We establish the connection with the entropy function in the literature [11,12]. To do so, we write the Lagrangian of the original action (4.4) in a suggestive form:

$$
\mathcal{L}\left(F_{0 m}^{I}, F_{m}^{I}, X^{a}, z^{\alpha}\right)
$$

Here $X^{a}$ is the collection of dynamical fields in the metric: $U$ for the static metric (2.1), while in the case of the non-static metric (3.1) it is given by $U$ and $\omega_{m}$. Note that we have to consider the three-dimensional metric $\gamma$ as background.

From the definition of $G_{I \mu \nu}$, or equivalently, from (4.3), we see that

$$
G_{I 0 m}=(\sqrt{\gamma})^{-1} \gamma_{m n} \frac{\partial \mathcal{L}}{\partial F_{n}^{I}}, \quad G_{I m}=(\sqrt{\gamma})^{-1} \gamma_{m n} \frac{\partial \mathcal{L}}{\partial F_{0 n}^{I}} .
$$

Then (4.4) and (4.5) show that the effective Lagrangian we obtained can be written as

$$
\mathcal{L}_{\text {eff }}\left(F_{m}^{I}, G_{m}^{I}, X^{a}, z^{\alpha}\right)=\mathcal{L}\left(F_{m}^{I}, F_{0 m}^{I}, X^{a}, z^{\alpha}\right)-\sqrt{\gamma} F_{0 m}^{I} G_{I}^{m}-\frac{1}{2} \sqrt{\gamma} R(\gamma) .
$$

The last term can be considered as a constant because $\gamma$ is not a dynamical field. Apart from this term, (4.8) clearly takes the form of a Legendre transform, where we switch from a description in $F_{m}^{I}, F_{0 m}^{I}$ (with gauge vectors $A_{\mu}^{I}$ ) to $F_{m}^{I}, G_{m}^{I}$ (with gauge vectors $A_{m}^{I}, A_{I m}$ ). By general principles of Legendre transformations this proves again that the field equations for $X^{a}$ and $z^{\alpha}$ of $\mathcal{L}_{\text {eff }}$ and of $\mathcal{L}$ are identical.

We can now carry this through in the spherically symmetric case. When we use the parametrization of the metric as in (2.28), and use the solution discussed in section [2.3, the only nonzero components of the field strengths $F_{m}^{I}$ and $G_{I m}$ are

$$
F_{\tau}^{I}=p^{I}, \quad G_{I \tau}=q_{I}
$$

After integrating out the angular dependence, (4.8) becomes the entropy function in the literature, derived from the 2-derivative Lagrangian (4.3). 


\section{Concluding remarks}

In this paper, we started from the nicely covariant action (2.2), describing Einstein-Maxwell theory for a number of Abelian gauge vectors. We investigated the field equations for stationary solutions. In particular, we showed that the field equations can be reproduced from an action that lacks in covariance, but makes electric/magnetic duality manifest. The key idea was to shift from four-dimensional gauge vectors $A_{\mu}^{I}$ to three-dimensional ones: $A_{m}^{I}, A_{I m}$. This effective action is given by

$$
S_{\text {eff }}=\int \mathrm{d} t \int \mathrm{d}^{3} x \sqrt{\gamma}\left[-\partial_{m} U \partial^{m} U-G_{\alpha \bar{\beta}} \partial_{m} z^{\alpha} \partial^{m} \bar{z}^{\bar{\beta}}+\frac{1}{8} \mathrm{e}^{4 U} \Omega_{m n} \Omega^{m n}\right]+\int \mathrm{d} t \int \mathrm{d}^{3} x \mathcal{L}_{\text {eff }}^{(1)},
$$

with

$$
\begin{aligned}
& \mathcal{L}_{\text {eff }}^{(1)}=\frac{1}{2} \sqrt{\gamma}\left(F^{I m} G_{I 0 m}-G_{I}^{m} F_{0 m}^{I}\right) \\
& =-\frac{1}{2} \frac{\mathrm{e}^{2 U}}{\mathrm{e}^{4 U} \omega^{2}-1}\left[\sqrt{\gamma}\left(\mathrm{e}^{4 U} \omega^{m} \omega^{n}-\gamma^{m n}\right)\left(\begin{array}{ll}
F_{m}^{I} & G_{I m}
\end{array}\right) \mathcal{M}\left(\begin{array}{c}
F_{n}^{J} \\
G_{J n}
\end{array}\right)+2 \mathrm{e}^{2 U} \omega_{m} G_{I n} F_{p}^{I} \varepsilon^{m n p}\right] .
\end{aligned}
$$

$F_{m}^{I}$ and $G_{I m}$ are given by (2.14). This action is actually a Legendre transformation of the spacetime covariant supergravity action (2.2) on the level of the (three-dimensional) gauge fields. This can be seen from equations (4.7) and (4.8). From the original Einstein equations, we see that we have to supplement the effective action with a set of constraints.

The result can be split up. In the static case, we have established the connection with the black hole potential, a concept which we can now extend to non-spherically symmetric setups. The constraints reduce to one equation, given in (2.32). Also we saw that the Legendre transform reduces to the entropy function in the literature, after eliminating the vector fields from the effective action. In the non-static case, we have found the same electric/magnetic duality invariant action of [9]. The new results we obtain are twofold. On the one hand, we have the new feature of seeing the effective action as a Legendre transform. On top of that we have now found the constraints (3.19), stemming from the original Einstein equations. They have to be supplied to the effective action when one considers non-supersymmetric setups and were not calculated before.

We hope that this analysis, in particular the explicit form of the constraints for the non-static effective action, can be a helpful step towards (not necessarily supersymmetric) stationary black hole solutions. Indeed, these constraint are automatically fulfilled for supersymmetric solutions, see for instance [19], but have to be taken into account when one wants to solve the system in absence of supersymmetry.

\section{Acknowledgments.}

We are grateful to Jan De Rydt, Frederik Denef, Renata Kallosh and Dieter Van den Bleeken for interesting and very useful discussions. We would also like to thank Oscar Maciá, who contributed to the early stages of this work. 
This work is supported in part by the European Community's Human Potential Programme under contract MRTN-CT-2004-005104 'Constituents, fundamental forces and symmetries of the universe', by the Federal Office for Scientific, Technical and Cultural Affairs through the 'Interuniversity Attraction Poles Programme - Belgian Science Policy' P6/11$\mathrm{P}$, and in part by the FWO - Vlaanderen, project G.0235.05. B.V. is Aspirant FWOVlaanderen. 


\section{References}

[1] S. Ferrara, R. Kallosh and A. Strominger, N=2 extremal black holes, Phys. Rev. D52 (1995) 5412-5416, hep-th/9508072

[2] A. Strominger, Macroscopic Entropy of $N=2$ Extremal Black Holes, Phys. Lett. B383 (1996) 39-43, hep-th/9602111

[3] S. Ferrara and R. Kallosh, Supersymmetry and Attractors, Phys. Rev. D54 (1996) 1514-1524, hep-th/9602136

[4] S. Ferrara, G. W. Gibbons and R. Kallosh, Black holes and critical points in moduli space, Nucl. Phys. B500 (1997) 75-93, hep-th/9702103

[5] P. K. Tripathy and S. P. Trivedi, Non-supersymmetric attractors in string theory, JHEP 03 (2006) 022, hep-th/0511117

[6] K. Goldstein, N. Iizuka, R. P. Jena and S. P. Trivedi, Non-supersymmetric attractors, Phys. Rev. D72 (2005) 124021, hep-th/0507096

[7] R. Kallosh, N. Sivanandam and M. Soroush, The non-BPS black hole attractor equation, JHEP 03 (2006) 060, hep-th/0602005

[8] G. W. Moore, Arithmetic and attractors, hep-th/9807087

[9] F. Denef, Supergravity flows and D-brane stability, JHEP 08 (2000) 050, hep-th/0005049

[10] B. Bates and F. Denef, Exact solutions for supersymmetric stationary black hole composites, hep-th/0304094

[11] A. Sen, Black hole entropy function and the attractor mechanism in higher derivative gravity, JHEP 09 (2005) 038, hep-th/0506177

[12] G. L. Cardoso, B. de Wit and S. Mahapatra, Black hole entropy functions and attractor equations, JHEP 03 (2007) 085, hep-th/0612225

[13] D. A. Rasheed, Non-linear electrodynamics: Zeroth and first laws of black hole mechanics, hep-th/9702087

[14] G. W. Gibbons, R. Kallosh and B. Kol, Moduli, scalar charges, and the first law of black hole thermodynamics, Phys. Rev. Lett. 77 (1996) 4992-4995, hep-th/9607108

[15] K. P. Tod, All metrics admitting supercovariantly constant spinors, Phys. Lett. B121 (1983) 241-244

[16] K. P. Tod, More on supercovariantly constant spinors, Class. Quant. Grav. 12 (1995) 1801-1820 
[17] X. Bekaert and M. Henneaux, Comments on chiral p-forms, Int. J. Theor. Phys. 38 (1999) 1161-1172, hep-th/9806062

[18] M. Henneaux and C. Teitelboim, Dynamics of (selfdual) p-forms, Phys. Lett. B206 (1988) 650

[19] G. Lopes Cardoso, B. de Wit, J. Kappeli and T. Mohaupt, Stationary BPS solutions in $N=2$ supergravity with $R^{2}$ interactions, JHEP 12 (2000) 019, hep-th/0009234 